\title{
Section VIII
}

\section{Fred Whipple Reflects}




\title{
THE FOREST AND THE TREES
}

\author{
FRED L. WHIPPLE \\ Smithsonian Astrophysical Observatory \\ 60 Garden Street \\ Cambridge, Massachusetts 02138 U.S.A.
}

\begin{abstract}
Some 24 points are made concerning the author's understanding of the cometary nucleus as developed in this colloquium. Comparisons with an earlier report are noted, and most of the earlier advances in concept are substantiated. The mean density of the Halley nucleus may have been underestimated. The dust/gas ratio apparently needs to be increased by as much as two times, perhaps to unity or higher. The nature of the rotation remains puzzling. CHON grains now appear to be more important sources of gas than thought earlier. A very low temperature at, and subsequent to, the formation of the nucleus continues to receive support, as does a solar-system composition. The ${ }^{12} \mathrm{C} /{ }^{13} \mathrm{C}$ ratio may, however, be lower than expected.

This paper also presents problems and data relevant to the origin and aging of comets. Do the short-period comets have a different source than the long-period ones? Among the long-period comets, the data support their aging statistically as the periods decrease. Criteria for the ages of short-period comets are much needed. Data and discussions then center on the orientation of cometary axes with respect to the galaxy and the ecliptic for longer period comets. The physical properties of comets in clusters defined by these axes differ and are of potential interest with regard to the origin of both short- and long-period comets.
\end{abstract}

\section{Introduction}

The title of this paper developed from my watching trees floating down toward the mouth of the Amazon River. Imagine an explorer who can study these floating trees and from this study alone must deduce the place and nature of the forest or forests from which they were uprooted. In analogy, where and how did our observed comets come into being?

As I was asked to make some comments about this colloquium, I have divided this paper into three parts: comments on the nature of comet nuclei, some problems regarding cometary origin, and some correlations among cometary data. The first part of the paper can only be sketched in character because of the finite space available to report on so many important contributions. I regret that a number of these contributions will be omitted. The third part of the paper contains correlations that are primarily suggestions of possibilities, because the data are insufficient to support most of these correlations as valid. 


\section{Comments on the Nature of Comet Nuclei}

\subsection{GENERAL REMARKS}

At the symposium on the diversity and similarity of comets held in Brussels in 1987 (see European Space Agency publication SP-278), I presented some 19 comments concerning cometary nuclei. These comments were based on previous cometary lore augmented by the results from the space missions to Halley's comet and by other international research efforts on comets accelerated by the 1986 apparition of P/Halley. Here I shall list these comments with corrections and alterations induced by subsequent research and particularly by presentations at this Bamberg Colloquium. Most of the original comments have foundations in references that are included in a summary paper on "Comets in the Space Age" (Whipple 1989).

These original abbreviated comments about cometary nuclei follow as headings, augmented by explanatory notes or documented alterations expressing my personal point of view following the Bamberg Colloquium.

\subsection{THE NUCLEUS}

2.2.1. A Discrete Nucleus. The VEGA and Giotto space missions to P/Halley have confirmed this fact.

2.2.2. A Black Nucleus, With a Very Low Albedo. This fact has been confirmed by the space missions and many infrared observations. The geometric albedo appears to range from a minimum that is perhaps as low as 0.02 to values that are perhaps as high as 0.10 to 0.15 for large, distant comets, with a typical value being probably on the order of about 0.04 for comets more or less $1 \mathrm{AU}$ from the Sun.

2.2.3. Low Density, $\rho \sim 0.1$ to $0.4 \mathrm{~g} \cdot \mathrm{cm}^{-3}$. However, some investigators would raise this value to about 0.8 to $2.0 \mathrm{~g} \cdot \mathrm{cm}^{-3}$ for $\mathrm{P} /$ Halley. I suspect that the value is more like 0.4 to $0.6 \mathrm{~g} \cdot \mathrm{cm}^{-3}$ for $\mathrm{P} / \mathrm{Halley}$, but that it is much lower for small, new comets and is perhaps $\sim 1 \mathrm{~g} \cdot \mathrm{cm}^{-3}$ for the nuclei of old, large short-period comets.

2.2.4. Increased Dimensions. Comets generally have increased dimensions as compared with the dimensions from previous calculations that were based on intrinsic magnitudes at large solar distances, when the comets were inactive (?) and not surrounded by clouds of particles (?).

2.2.5. Prolate Shape. The space missions have clearly demonstrated the irregular "potato" or "peanut" shape of $\mathrm{P} / \mathrm{Halley}$, while the light curves of distant comets, particularly the light curves by Jewitt and Meech (see Jewitt, this volume), show that such shapes (i.e., not rough spheroids) are probably also typical for distant, black-reddish asteroids.

2.2.6. Ultra-Fine Dust $\left(<10^{-13} \mathrm{~g}\right)$. The space missions to $\mathrm{P} /$ Halley have shown the presence of dust down to $\sim 10^{-16} \mathrm{~g}$ in size, too small to scatter light effectively and hence not previously observed. 
2.2.7. CHON Dust. The space missions have demonstrated that $\mathrm{P} / \mathrm{Halley}$ dust contains a sizable fraction that is composed of the relatively abundant light elements $\mathrm{C}, \mathrm{H}, \mathrm{O}$, and $\mathrm{N}$. Apparently, CHON carries a considerable fraction of the rarer volatiles (see Section 2.2.13). Huebner et al. (this volume) show that the hydrocarbon POM (polyoxymethylene, $\mathrm{nH}_{2} \mathrm{CO}$, a long polymer chain) is carried by the CHON, while Jackson (this volume) shows that $\mathrm{C}_{2}$ arises similarly (probably from acetylene, $\mathrm{C}_{2} \mathrm{H}_{2}$, via $\mathrm{C}_{2} \mathrm{H}$ ). Mukai (1989) finds the CHON grains to be hotter than the silicate grains. Jessberger and Kissel (this volume) find the CHON grains' density to be $\sim 1.0 \mathrm{~g} \cdot \mathrm{cm}^{-3}$.

2.2.8. A Poorly Known Gas/Dust Ratio. The gas output of $\mathrm{P} /$ Halley is well-determined, and most investigators have placed the ratio at $\sim 1.2$, though Jessberger and Kissel have placed it at 0.9 . However, Szegö (this volume) has found evidence for glitches, caused by impacts, in Giotto's motion, leading McDonnell, Lamy, and Pankiewicz (this volume) to credit dust with twice the mass abundance of gas $(2 \pm 1)$. The presence of sizable particles around some comets has been shown by radar (Campbell, Harmon, and Shapiro 1989), while West (1990) finds that P/Halley shows an asymmetric coma outbound at greater than $10 \mathrm{AU}$ from the Sun.

\subsubsection{Composition Like Type 1 Carbonaceous Chondrites; Lack of C in Gas Now Found} in Dust. Delsemme (this volume), however, believes Fe to be deficient in $\mathrm{P} / \mathrm{Halley}$ (by a factor of 2) and Si to be similarly overabundant, which he attributes to thermal processes in the solar nebula. Otherwise, the elemental abundances appear to be satisfactorily equivalent to the solar-system abundances. Hanner and Tokunaga (this volume) find evidence for olivine grains in the infrared (IR).

2.2.10. Solar-System Isotopes Ratios for ${ }^{34} \mathrm{~S}^{32} \mathrm{~S}$ and $\mathrm{D} / \mathrm{H}$. The ratios for ${ }^{14} \mathrm{~N} / 1 \mathrm{~N}^{15}$ have also been confirmed in a review by Vanysek (this volume). The ratio for ${ }^{12} \mathrm{C} /{ }^{13} \mathrm{C}$ may, however, be uncomfortably low, at $\sim 65$ (not $\sim 85$ ), according to Wehinger (personal communication).

2.2.11. Active Areas $<10 \%$ Even for $P /$ Halley. The evidence now suggests that periodic comets are active only over extremely small percentages of their surfaces. How about "new" comets and disappearing comets?

2.2.12. Thick, Insulating Mantle (High T). A thick, insulating mantle found for $\mathrm{P} / \mathrm{Halley}$, coupled with small active areas, suggests that there is a crust of deep crevasses, allowing the effects of wasting, slides, etc. that precipitate outbursts. Perhaps it is even a source of splitting?

\subsubsection{3. $\mathrm{H}_{2} \mathrm{O}$ Is the Most Prominent Gas, But More Active Material ( $\mathrm{CO}_{2}$ ?) Is Present.} Consider Comet Bowell, a new comet with a large coma when the comet was discovered at $>7$ AU from the Sun. However, Delsemme (1988) considers formic acid (HCO-OH) and formaldehyde $\left(\mathrm{H}_{2} \mathrm{CO}\right)$ to be slightly more abundant than $\mathrm{CO}_{2}$ in the gas from $\mathrm{P} / \mathrm{Halley}$ $\left(\sim 4 \%\right.$ each, as compared with $80 \%$ for $\left.\mathrm{H}_{2} \mathrm{O}\right)$, with a large amount of CO evolving from the former two gases and the total amounting to $\mathrm{CO} / \mathrm{H}_{2} \mathrm{O}=13 \pm 3 \%$. See also Eberhardt et al. (1986). 


\subsubsection{Colors and Albedos Like Those of Far-Out Asteroids (e.g., Trojans).}

2.2.15. Slow Rotation Values (Slower Than Whipple's Values). Feldman (this volume) shows that variation in the Lyman alpha image is not a reliable criterion for the rotation of a comet nucleus. Belton (this volume) makes a strong argument that for P/Halley, the 2.2day period and 7.4-day Euler nodding are not acceptable. The subject of $\mathrm{P} / \mathrm{Halley}$ 's rotation period remains controversial.

2.2.16. $S_{2}$ Shows a Low T From Formation to the Present. This conclusion is supported by Weaver, Mumma, and Larson (this volume) from their measures of the ratio of the para to ortho water in $\mathrm{P} / \mathrm{Halley}$. They also suspect that the ratio differs between old and new comets. Klinger (this volume) notes that $\mathrm{CO}_{2}$ could also be amorphous and that amorphous ice can trap and hold atoms and molecules if $\mathrm{T}<77 \mathrm{~K}$. Bar-Nun (personal communication) stresses the importance of observing noble gases as measures of $\mathrm{T}$ conditions during formation and afterwards.

2.2.17. Greenberg Interstellar Process. This process is favored as the method of origin of icy mineral grains.

2.2.18. Halley Nucleus Looks Battered (Made of Many 1-km Cometesimals?). Investigators have found a large variation in the composition over the surfaces of different comets. Weaver, Mumma, and Larson (this volume) have found the formaldehyde abundance to vary over Comet Halley, while Meech (this volume) has found $\mathrm{CO}^{+}$to be more prevalent in Comet Wilson than in Comet Halley, and Feldman (this volume) has found $S_{2}$ to be the most prevalent in a burst of Comet IRAS-Araki-Alcock.

2.2.19. Probably Formed in the Solar Nebula. But where-the cloud or the disk? Criteria to tell the differences have yet to be developed. Perhaps the basic temperature will be the clue?

2.2.20. The Night Side of P/Halley. Gombosi (this volume) has shown how the spreading of the jet action could account for dust on the night side without activity there.

2.2.21. Theories of Comet Activity Are Many, With Major Differences. Crifo, Horanyi and Mendis, Huebner et al., Jessberger and Kissel, and Jackson have all presented somewhat differing viewpoints in this volume.

2.2.22. Solar Nongravitational Force. Yeomans (this volume) treats solar radial nongravitational force as the prime factor, so that the effect depends largely upon the comet's light curve rather than upon the orientation of its pole.

2.2.23. Lightning? I note, to my surprise, that no one has reported any effects of lightning measured in the Halley missions, although lightning should be prevalent in swarms of dust, ions, and electrons immersed in electromagnetic fields. 
2.2.24. A Vital Requirement for the Future. Rendezvous missions and/or other more sophisticated missions to comets are vitally needed.

\section{Some Problems Regarding Cometary Origin}

Four sources of the observed comets are being considered seriously:

(a) Members of the Oort (1950) cloud originating in the Uranus-Neptune region and later thrown out of it.

(b) Members of the Oort cloud aggregated originally from the solar nebula during the collapse (see, e.g., Hills 1982).

(c) Comets captured from the interstellar medium.

(d) Comets formed in the collapsed solar nebular disk beyond Neptune and Pluto (the "Kuiper belt") and somehow perturbed into short-period orbits.

The capture of interstellar comets, even though they may be numerous in space, appears so unlikely that this source (c) is discounted by most students of the subject.

The "classical" idea (a) of cometary origin in the region of the planets seems to be losing its appeal as the evidence grows for extremely low temperatures during and since formation. Delsemme (1988) holds, however, that the diversity of the grains in comets requires just such a region in which a mixture of grains could have occurred. Everhart (1977) concluded that Oort-cloud comets with low inclinations and perihelia in the planetary region could eventually (with enormous losses) be perturbed into short-period orbits. Duncan, Quinn, and Tremaine (1990), however, find that Oort-cloud comets, so perturbed from a "spherical population," would contribute short-period orbits with much greater orbital inclinations than those observed. On the other hand, they find that disk comets in low-inclination orbits as defended by Fernandez (1980 and, with Ip, 1983) will provide the short-period comets in low-inclination orbits without the prodigious losses arising in Everhart's calculations.

Thus we seem to be left with short-period comets that may have been formed in the protosolar disk beyond the outermost planets in a rather cold region, while the nearly parabolic and long-period comets may have originated in the outer-planetary region, relatively warm, or collapsing with the solar nebula at very low temperatures more typical of the interstellar clouds.

Can we, then, find any distinguishing physical characteristics of comets to indicate their source? In the search, we are confused by disturbing factors arising between origin and our observations from environment and also by factors depending solely upon cometary dimensions. As we have noted, each cometesimal in a comet nucleus may have experienced a somewhat individual history, while its velocity of impact may have greatly affected the volatility and cohesiveness of the shattered "matrix" material that it has contributed. The matrix may not have "sintered" enough to be as physically strong as the basic cometesimals and may also be less volatile. Hence, it may blow out more easily in the form of relatively large grains and hunks, but be less volatile and active than the cores of cometesimals.

"New" comets may show unusual activity at large solar distances, because of cosmic-ray-induced volatility (C/Kohoutek 1973 XII) or because of a blow-off of an outer matrix or grain layer (C/Bowell $1982 \mathrm{I})$. Large, old comets may possess a radioactively 
heated core of $\mathrm{H}_{2} \mathrm{O}$ ice and grains and even packets of more volatile ices that are condensed in their outer shells and that are eventually exposed.

Hence, among comets of a common origin, we may observe confusing physical characteristics that might deceive us into classifying them as being different in basic nature or source. Typical variants to be expected among active comets include:

(a) For "new" comets, an outer frosting of highly volatile material, amorphous in nature.

(b) The blowing-off of thick grain mantles by "new" comets, after the comets have previously been exposed to hot stars or supernovae.

(c) A typical mix of cometesimals and matrix with or without variation in composition, depending upon the position of the source on the nucleus.

(d) Only shattered matrix.

(e) Only cometesimals.

(f) $\mathrm{H}_{2} \mathrm{O}$ ice and grains only, from the radioactively heated cores of old comets.

(g) $\mathrm{H}_{2} \mathrm{O}$ ice almost alone.

(h) More volatile ices than $\mathrm{H}_{2} \mathrm{O}$ generally blowing out matrix grains.

Since the ground-breaking work by Oort and Schmidt (1951) suggesting systematic differences in the light curves between "new" and "old" comets, several investigators have studied the subject. Donn (1977) found no correlation among the spectra of 85 comets for a statistical difference in the ratio of continuum to emission for new and "evolved" comets. Nor did Kresak (1977) find a difference in the light curves. On the other hand, Kresak $(1973,1977)$ did find that short-period comets are rarely seen beyond $4 \mathrm{AU}$, with a sharp break near $3 \mathrm{AU}$, while comets with periods greater than 2,000 yr are frequently observed at far greater solar distances, an effect ascribed to $\mathrm{H}_{2} \mathrm{O}$ ice in older comets versus more volatile ices in younger comets. This effect is supported by the observation of Marsden and Sekanina (1973) that new comets constitute such a large fraction of long-period comets with large perihelion distances (presumably the effect of thin, highly active layers on the surface of new comets, making them brighter at their first appearance than they are subsequently).

Meisel and Morris (1976) carefully analyzed photometric data for more than 100 comets and related the exponent $n$ in the solar radial component of brightness, $r-n$, to the "age" of the comet. They found a larger correlation with perihelion distance than with preand post-perihelion means of $n$ for new and old comets. In other words, they found only an extremely weak confirmation of the Oort-Schmidt conclusion. In a somewhat similar study of more than 100 cometary light curves, Whipple (1978) found the exponent $n$ to be significantly smaller for new comets than for older comets before perihelion, but statistically the same after perihelion for all comets of periods $>25 \mathrm{yr}$. For comets of shorter periods, $n$ tends to be larger and erratic. Thus, the early indications by Oort and Schmidt appear to be fairly well confirmed.

In recent years, a number of observational studies have failed to detect real compositional differences between new and old comets (e.g., A'Hearn and Millis 1980; Weaver et al. 1981; Newburn and Spinrad 1984, 1985, 1989; Cochran 1987). As for aging, the period of a long-period comet is some measure, at least statistically, of the time since the comet was perturbed into an orbit with a small perihelion distance. For shortperiod comets, unfortunately, there exists no method for dating their capture. At best, we 
Table 1. Nongravitational Forces vs. Dust/Gas

\begin{tabular}{|c|c|c|c|c|c|}
\hline \multicolumn{6}{|c|}{ Period > $1,000 \mathrm{yr}$} \\
\hline Dust/Gas & $N_{o}$ & $<q>_{\mathrm{AU}}$ & $<A_{1}>$ & $\left\langle\left|A_{2}\right|\right\rangle$ & $\left.<\left|A_{2}\right|\right\rangle_{\text {Max }}$ \\
\hline $\begin{array}{l}\text { High } \\
\text { Medium and Low }\end{array}$ & $\begin{array}{l}4 \\
3\end{array}$ & $\begin{array}{l}0.77 \\
0.71\end{array}$ & $\begin{array}{l}1.9 \\
3.8\end{array}$ & $\begin{array}{l}0.15 \\
0.60\end{array}$ & $\begin{array}{l}-- \\
--\end{array}$ \\
\hline \multicolumn{6}{|c|}{ Period $<1,000 \mathrm{yr}$} \\
\hline Dust/Gas & $N_{o}$ & $<q>_{\mathrm{AU}}$ & $<A_{1}>$ & $\left\langle\left|A_{2}\right|\right\rangle$ & $\left.<\left|A_{2}\right|\right\rangle_{\text {Max }}$ \\
\hline $\begin{array}{l}\text { High } \\
\text { Medium } \\
\text { Low }\end{array}$ & $\begin{array}{l}3 \\
4 \\
2\end{array}$ & $\begin{array}{l}1.32 \\
1.03 \\
0.96\end{array}$ & $\begin{array}{l}0.21 \\
0.60 \\
?\end{array}$ & $\begin{array}{l}0.014 \\
0.045 \\
0.032\end{array}$ & $\begin{array}{l}0.016 \\
0.056 \\
0.062\end{array}$ \\
\hline
\end{tabular}

may be able to trace back an orbit to a close planetary approach, whereupon the earlier history becomes indeterminate. Similarly, a study of a derived meteor stream may give evidence of limited recent history. Although Kresak (1987) lists 10 observable phenomena associated with aging, none (except meteor streams) carry any mark of time.

\section{Some Correlations Among Cometary Data}

\subsection{DUST/Gas Ratio AND NONGRAVITATIONAL FORCES}

Although Donn (1977) found no correlation between the dust/gas ratio in new and "evolved" comets, his data do suggest correlations with nongravitational (NG) effects on the orbits. Table 1 lists the following in successive columns: his value of the dust/gas (ratio); $N_{o}$ (comets); $\langle q\rangle_{\mathrm{AU}}$ (mean perihelion distance); $\left\langle A_{I}\right\rangle$ (mean radial $N G$ acceleration); $\left|A_{2}\right|$ (mean absolute tangential NG acceleration); and $\left\langle\left|A_{2}\right|\right\rangle_{\mathrm{Max}}$ (maximum observed $\left|A_{2}\right|$ ), where the dust/gas ratios are Donn's values and $A_{1}$ and $A_{2}$ are taken from and defined in Marsden's (1986) catalogue of cometary data. Values of these quantities are listed in successive lines for seven comets with periods $>1,000 \mathrm{yr}$ and nine comets with periods $<1,000 \mathrm{yr}$.

The numbers in Table 1 suggest three conclusions: 
(a) For all types of comets, NG accelerations are smaller for comets with high values of dust/gas.

(b) In each dust/gas category, NG accelerations are larger for comets with $\mathrm{P}>1,000 \mathrm{yr}$ than for those with $\mathrm{P}<1,000 \mathrm{yr}$.

(c) The effects of (a) and (b) are affected little, if any, by the perihelion distances of the comets.

We may assume that the comets with shorter periods are older statistically than those with longer periods. Hence, the data suggest the physical result that the older comets are less active by an order of magnitude than the younger comets in terms of NG forces and, therefore, in terms of gas ejection. This further implies the successive development of grainy mantles as comets age. Despite the paucity of data, the effects appear to be so strong as to invite serious consideration.

\subsection{COMETARY STATISTICS OF MAGNITUDE VARIATIONS}

This section involves the reanalysis of the cometary magnitude variations published by Meisel and Morris (1976), particularly their derived values of $n$, the exponent in $r^{-n}$ for the magnitude equation mentioned earlier ( $r=$ the solar distance in AU). Because $n$ varies so erratically from comet to comet, great weights need not be used for extremely long series of observations. The value of $n$ should only be fairly well determined. Hence, I have adopted a weight of 1.0 for all values of $n$ determined from 100 or more observations and a weight equal to the number of observations divided by 100 for lesser numbers of observations, down to weight $=0.10$, below which the $n$ value is neglected. This choice of weighting broadens the range of input to more comets without weakening the accuracy of $n$ for the average contributing comet.

The age classification was taken as:

$$
\begin{array}{ll}
\text { I (new) } & 1 / a \times 10^{+6} \leq 50(\mathrm{AU})^{-1} ;(P)>2.8 \times 10^{6} \mathrm{yr} \\
\text { II } & 5 \times 10^{-5}<1 / a<0.00215(\mathrm{AU})^{-1} ; 10^{4}<P<2.8 \times 10^{6} \mathrm{yr} \\
\text { III } & 0.00215<1 / a<0.01(\mathrm{AU})^{-1} ; 10^{3}<P<10^{4} \mathrm{yr} \\
\text { IV } & 0.01<1 / a<0.117(\mathrm{AU})^{-1} ; 25<P<10^{3} \mathrm{yr}
\end{array}
$$

where $P$ is the period. For the longer period comets, $1 / a$ original values were taken from the compilations of Marsden, Sekanina, and Everhart (1978), and Everhart and Marsden (1983, 1987).

In each age group, the mean values of $n$ and $a$ were determined from the tables of Meisel and Morris (1976) separately for the "Pre-Perihelion Dominated Solution," the "Post-Perihelion Dominated Solution," and their "Combined Pre- and Post-Perihelion Periods." The results of these calculations are presented in Table 2 , where $\langle q\rangle$ is the weighted mean perihelion distance, $\langle n\rangle$ is the weighted mean of $n$, and $\sigma(n)$ is the mean error of $\langle n\rangle$.

Perusal of Table 2 shows immediately that the mean values of $q$ vary considerably, while Meisel and Morris note that "the $n$ values do not show a significant correlation." For both the pre- and post- $q$ data, $\langle n\rangle$ increases strongly with comet age (i.e., with class or inversely with period). The combined pre- and post- $q$ data are mixed. Also, the post- $q$ values of $\langle n\rangle$ are consistently greater than the pre- $q$ values. These results are largely 
Table 2. Aging of Longer Period Comets

\section{Mean Values of $\bar{n}$}

\begin{tabular}{lcccc}
\hline Orbit Class & I & II & III & IV \\
\hline Maximum Period (yr) & $\infty$ & $2.8 \times 10^{6}$ & $10^{4}$ & $10^{3}$ \\
Minimum Period (yr) & $2.8 \times 10^{6}$ & $10^{4}$ & $10^{3}$ & 25
\end{tabular}

\section{Pre-Perihelion Dominated}

\begin{tabular}{|lllll|}
\hline Number of Comets & 10 & 5 & 5 & 6 \\
\hline
\end{tabular}

$\begin{array}{lrrrr}\langle q\rangle(\mathrm{AU}) & 0.43 & 0.90 & 1.17 & 0.71 \\ \langle n\rangle & 2.45 & 3.11 & 3.32 & 3.83 \\ \sigma(n) & \pm 0.35 & \pm 0.71 & \pm 0.52 & \pm 0.49\end{array}$

\section{Post-Perihelion Dominated}

\begin{tabular}{lrrrr}
\hline Number of Comets & 15 & 16 & 9 & 17 \\
& & & & \\
$<q>(\mathrm{AU})$ & 1.20 & 1.54 & 0.88 & 0.80 \\
$<n>$ & 3.16 & 3.87 & 4.48 & 4.94 \\
$\sigma(n)$ & \pm 0.26 & \pm 0.58 & \pm 0.53 & \pm 0.79
\end{tabular}

Combined Pre- and Post-Perihelion

\begin{tabular}{lrrrr|}
\hline Number of Comets & 10 & 8 & 5 & 2 \\
\hline & & & & \\
& & & & \\
& & & & \\
$\sigma(n)$ & \pm 0.54 & 2.39 & 5.05 & 3.3 \\
& & \pm 0.60 & \pm 1.86 & \pm 0.4 \\
\hline \hline
\end{tabular}


consistent with Whipple's (1978) conclusions, but, of course, the two data sets have much in common, since Meisel and Morris applied critical systematic corrections to the original observations. All in all, it appears that the long-period comets show aging in their light curves that is not just a "new"-versus-"old" relation, but is statistically a persistent aging on successive returns.

\subsection{SOLAR DISTANCES AT DISCOVERY AND AT LAST OBSERVATIONS OF COMETS}

Kresak (1977) has used the solar distances of discovery $\left(r_{l}\right)$ and last observations $\left(r_{2}\right)$ of comets to correlate with their ages. A somewhat different approach to the same end is presented here. For comets with perihelia from 1800 to 1900 , values of $r_{1}$ and $r_{2}$ were obtained from Vsekhsvyatskii's (1964) catalogue of comets, as were values of the comets' absolute magnitudes, $H_{10}$, defined as

$$
H_{10}=m-2.5 \log \left(\Delta^{2} r^{4}\right)
$$

where, as before, $m$ is the observed magnitude, $\Delta$ and $r$ are the geocentric and heliocentric distances in AU, and the exponent 4 in $r$ corresponds to $n=4$ in Section 4.3.

The selection of comets was limited to the time period of primarily visual observations, largely with comparable telescopes. The comets were divided into groups of increasing age: I, II, III, and IV as in Section 4.2, except that group IV was cut off at an orbital period of $200 \mathrm{yr}$ instead of $25 \mathrm{yr}$. As Kresak noted, the values of $r_{7}$ and $r_{2}$ are very sensitive to the cometary absolute magnitude. Hence, for each group, the relationship $\Delta r_{i} / \Delta m a g$ was obtained by a least-squares solution of $A_{i}$ and $B_{i}$ in the equation

$$
A_{i}+B_{i} H_{10}=r_{i}
$$

where $i=1$ or 2 .

Therefore, $B_{i}=\Delta r_{i} / \Delta$ mag can be used to correct $r_{i}$ from its mean value $<r_{i}>$ to a standard value, which is taken as $H_{10}=6.0$. Hence, the correction to $\left\langle r_{i}\right\rangle$ becomes $\left(\Delta r_{i} / \Delta m a g\right)\left(6.0-<H_{10}>\right)$, where $<H_{10}>$ is the mean value of the absolute magnitude for the group.

Table 3 presents the results of this effort in a form similar to that of Table 2 . The mean errors of the $r$ 's are not given in the table, but they are roughly $\pm 0.15 \mathrm{AU}$ for $\left\langle r_{i}\right\rangle$ and $\pm 0.30 \mathrm{AU}$ for $\left\langle r_{2}\right\rangle$. Comet Barnard $1889 \mathrm{I}$, a new comet, was omitted from the summation because of its extreme values $\left(r_{1}=2.55\right.$ and $\left.r_{2}=8.22 \mathrm{AU}\right)$, which would have unduly biased the averages in favor of new comets. The decrease in $\left\langle r_{1}\right\rangle$ and $<r_{2}>$ with age for the comets as seen in Table 3 is strongly suggestive, but not yet definitive.

\subsection{ON THE ORIENTATION OF COMETARY ORBITS}

The correlations discussed so far have involved only the aging of the long-period comets, providing strong evidence that the aging process continues beyond simply a "new"-versus-"old" type of criterion. R. Lüst (1984) has shown that the lines of apsides (or aphelia) of long-period comets tend to avoid a $20^{\circ}$ strip centered on the galactic equator and also the two polar regions from galactic latitudes $(b) \pm 60^{\circ}$; this has been confirmed by 
Table 3. Another Search for Aging Effects in Long-Period Comets

\section{Solar Distance of Discovery, $r_{I}$}

\begin{tabular}{|lllll|}
\hline Orbit Class & I & II & III & IV \\
\hline
\end{tabular}

Maximum Period (yr)

Minimum Period (yr)

$\begin{array}{cccc}\infty & 2.8 \times 10^{6} & 10^{4} & 10^{3} \\ 2.8 \times 10^{6} & 10^{4} & 10^{3} & 200\end{array}$

\begin{tabular}{|lllll|}
\hline Number of Comets & 8 & 21 & 25 & 15 \\
\hline
\end{tabular}

$\begin{array}{lrrrr}<r_{l}>_{\text {obs. }}(\mathrm{AU}) & 1.59 & 1.50 & 1.40 & 1.28 \\ <H_{10}> & 5.64 & 5.81 & 5.35 & 6.68 \\ <\Delta r_{1} / \Delta \text { mag }> & -0.14 & -0.14 & -0.15 & -0.15 \\ <r_{1}>_{\text {corr. }}(\mathrm{AU}) & 1.54 & 1.48 & 1.30 & 1.38\end{array}$

Last Observed Solar Distance, $r_{2}$

\begin{tabular}{lrrrr}
\hline Number of Comets & 8 & 22 & 24 & 14 \\
& & & & \\
& & & & \\
& 2.77 & 2.59 & 2.14 & 1.99 \\
$<H_{10}>$ & 5.64 & 5.81 & 5.35 & 6.68 \\
$<\Delta r_{2} / \Delta$ mag $>$ & -0.33 & -0.65 & -0.33 & -0.29 \\
$<r_{2}>$ corr. $(\mathrm{AU})$ & 2.65 & 2.47 & 1.98 & 2.08
\end{tabular}

Delsemme (1987). The explanation by Harrington (1985), Morris and Muller (1986), and Heisler and Tremaine (1986) appears to be correct: the density gradient normal to the galactic plane perturbs comets in the Oort cloud proportionally to a $\sin b \cos b$ law, the socalled "galactic tide" effect.

Biermann, Huebner, and Lüst (1983) have clearly established the reality of a cluster of aphelia of long-period comets in the region in galactic longitude, $180^{\circ} \leq l \leq 248^{\circ}$, and latitude, $-2^{\circ}<b<+38^{\circ}$ (the BHL cluster). The cluster fills in part of the depleted zone $\left(|b|<10^{\circ}\right)$ and strongly calls for a stellar passage that perturbed the cloud comets. Delsemme (1986) finds another striking cluster (D), quite elongated, passing through the ecliptic pole, with most of the members moving in much the same direction at aphelion. $\mathrm{He}$ attributes this cluster to the slow passage of a brown dwarf star. 


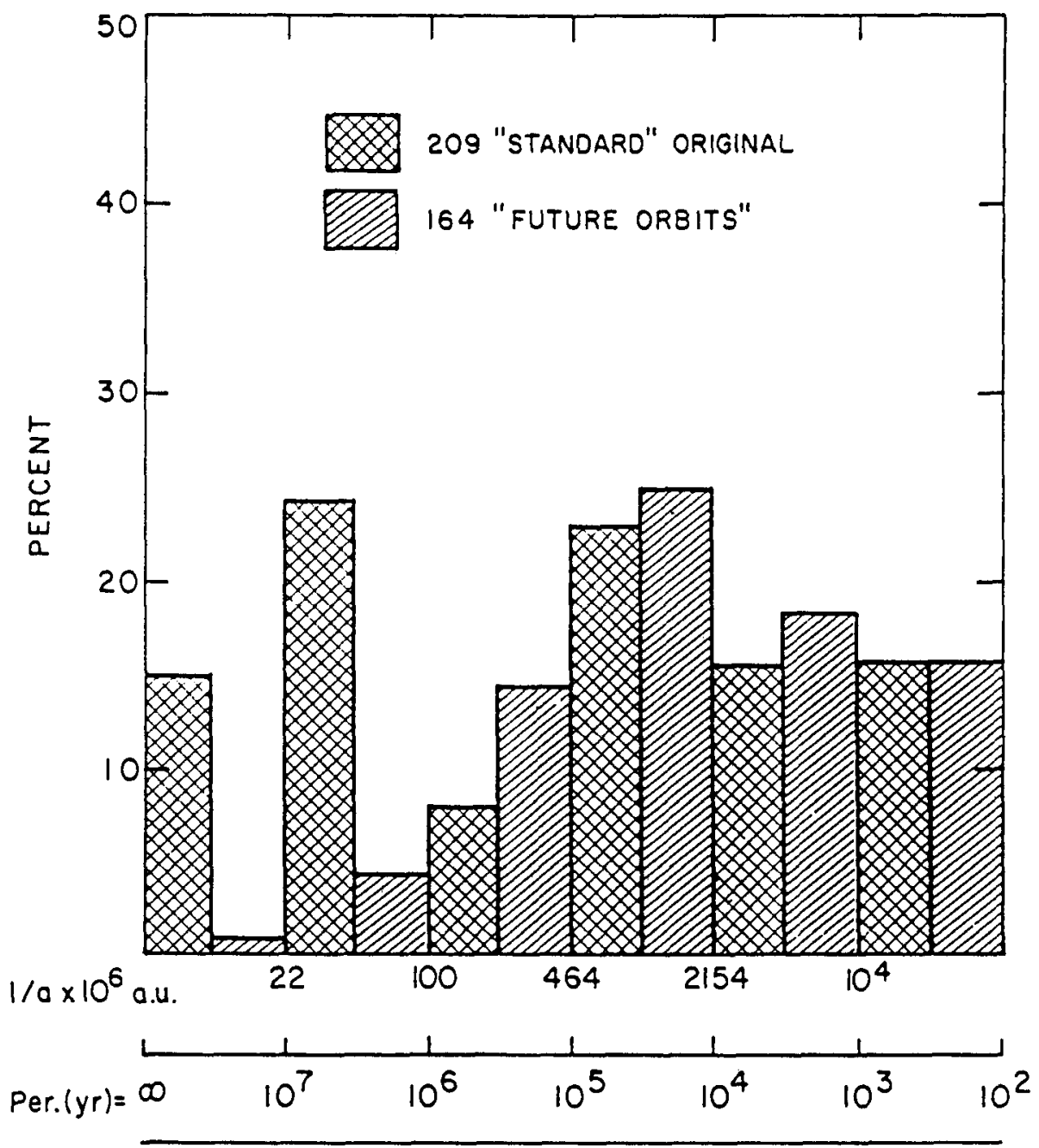

Figure 1. Original and future orbits of 209 long-period comets.

The populations of comets in terms of the galactic coordinates of their aphelia provide some possible clues about their origin and aging. To establish a base for comparison, 209 comets that have periods exceeding $100 \mathrm{yr}$ and that have been observed since 1900 are presented in Figure 1.

The block diagrams show percentage numbers versus original values of $1 / a(\mathrm{AU})^{-1}$ from Marsden and Sekanina (1973), Marsden, Sekanina, and Everhart (1978), Everhart and Marsden $(1983,1987)$, and Marsden (the 1986 catalogue, including the table of "ellip- 
tical orbits"). The corresponding percentage of "future" orbits of these comets compared with the "originals" is indicated in Figure 1, with $45(22 \%)$ having been lost from the solar system as the result of planetary perturbations.

The apparent dearth of comets with periods of a few hundred thousand years had, in the past, suggested to me that there may have been a dearth of new comets over a considerable interval of time in the past. Figure 1, however, suggests that the large nonGaussian distribution of $\Delta 1 / a$ induced by planetary perturbations may account for the dip. Essentially no new comets enter the inner solar system with such short periods. The figure clearly shows that a continuous contribution of new comets is essential to the maintenance of the distribution shown.

The population of the BHL cluster appears in Figure 2, showing the present distribution of 30 members observed since 1900, not including the sungrazers, and the return of 18 after losses (37\%) by planetary perturbations. Figure 3 shows the corresponding data for 29 members of the Delsemme cluster and the distribution of 21 possible returns ( $28 \%$ loss). The latter cluster has relatively fewer members with periods of less than $10^{4} \mathrm{yr}$, compared with the BHL cluster and the "standard" distribution (also shown in Figures 2 and 3). Although the numbers are scant, Figures 2 and 3 suggest that the D cluster is relatively newer, not having been active as long as the BHL cluster.

Delsemme stresses the important point that members of his cluster preponderantly have motions at aphelion that are "almost perpendicular to the plane of the ecliptic." The members of the BHL cluster, on the other hand, are moving almost perfectly at random when at aphelion. It is interesting to study the motions at aphelion of the 18 members of the peculiar group that have aphelia lying less than $10^{\circ}$ from the galactic plane and that are not members of the BHL cluster or sungrazers. The motion is within $30^{\circ}$ of the normal $( \pm)$ to the galactic plane for 12 of the 18 , with only one directed within $30^{\circ}$ (actually, 27.7) of a parallel to the plane. The group also has a peculiar distribution of periods, with half lying in the range of $10^{3}<P<10^{4} \mathrm{yr}$, only three lying in the range of $10^{4}<P<10^{5} \mathrm{yr}$, and only one that has a longer period (the apparently new, unusually dusty C/ArendRoland, 1957 III).

Seven members are of special interest, as they lie at the intersection of the galactic and ecliptic planes, all with ecliptic longitude, $L$, confined by $90^{\circ}<L<99^{\circ}$, and with relatively small ecliptic latitudes, $B$. Let us call them the GE group (see Table 4 ). Whether or not they are statistically significant as a group, they lie in a region where "galactic tides" should be ineffective. All have intermediate perihelion distances $(0.48<q<1.64 \mathrm{AU})$ and positive ecliptic motions $\left(13^{\circ}<i<79^{\circ}\right)$, but generally high ecliptic inclinations as compared with those of the short-period comets. The periods lie in three groups: 2 at 270 and $583 \mathrm{yr}, 4$ in the range of 1,398 to $2,800 \mathrm{yr}$, and 1 of the highest inclination, at $16,500 \mathrm{yr}$. The two extreme periods correlate directly with the orbital inclinations, with the other inclinations lying between these extremes, suggesting a reduction in inclination with period decrease. Four of the seven are among the six deviants from motion within $30^{\circ}$ of normal to the galactic plane. The group seems worthy of further study, both from a physical and an orbital point of view.

Physically, the BHL cluster demands attention because it contains the aphelia of P/Halley, C/Kohoutek 1973 XII, C/Morehouse 1908 III, two of the five comets with $100<P<200 \mathrm{yr}$, and, if slightly enlarged, the Kreutz sungrazers and ten of the 20 comets listed as "Great" in Marsden's catalogue (1986). On the other hand, the comets in the Delsemme cluster appear to be average, as do those in the GE group. It is true that the 


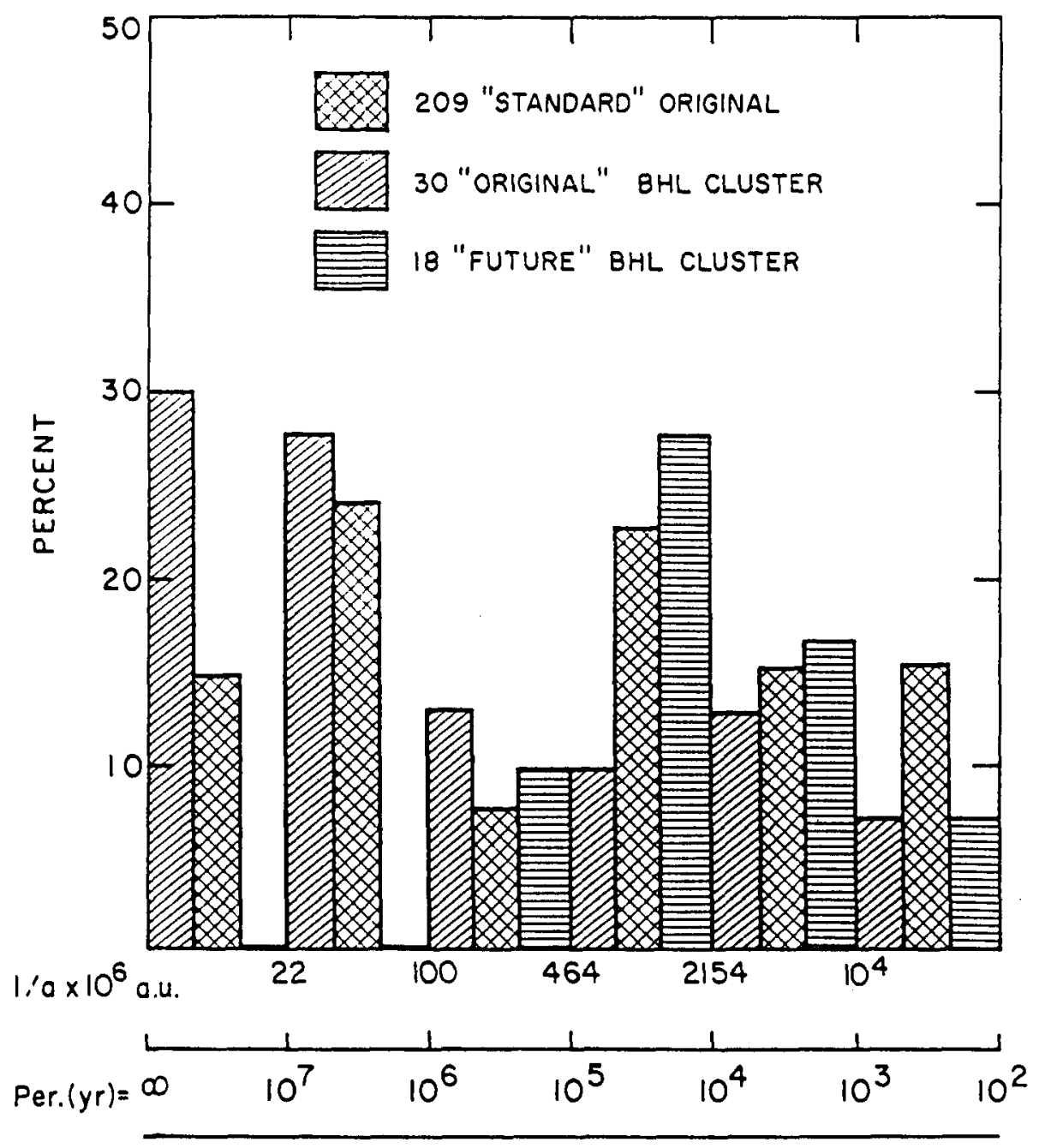

Figure 2. Original and future orbits of $30 \mathrm{BHL}$ cluster comets.

presence of Halley's comet, the sungrazers, and those comets of $100<P<200$ yr was probably the result of perturbations, not representations of their "original" axial orientations, but nevertheless, the question still remains as to whether different sources or origins may have been involved.

New comets of low inclination to the ecliptic are of especial interest, as they may represent the progenitors of the short-period comets. Of the 209 orbits of high accuracy 




Figure 3. Original and future orbits of $29 \mathrm{D}$ cluster comets.

studied in Figure 1 , only eight meet the requirements of $1 / a<100 \times 10^{-6}(\mathrm{AU})^{-1}$ and $i<35^{\circ}$. Of these eight, five are of extreme interest:

(a) Bowell $1982 \mathrm{I}$, which, when discovered at 7.3 AU, already had a welldeveloped disk of large grains, indicating activity at much greater solar distances (see, e.g., Sekanina 1982 and A'Hearn et al. 1984).

(b) Kohoutek 1973 XII, the notorious comet that was discovered near the orbit of Jupiter. 
Table 4. The GE Group

\begin{tabular}{|c|c|c|c|c|c|c|}
\hline Year & Name & $\stackrel{q}{\mathrm{AU}}$ & $\begin{array}{l}\text { 1/a(orig.), } \\
(\mathrm{AU})^{-1} \times 10^{6}\end{array}$ & $i$, deg. & $L$, deg. & $B$, deg \\
\hline 1846 I & de Vico & 1.48 & 5,131 & 47.4 & 97.3 & -16.0 \\
\hline 1863 IV & Tempel & 0.71 & 1,546 & 78.1 & 98.1 & -2.7 \\
\hline 1906 VII & Theile & 1.21 & 14,328 & 56.4 & 90.2 & +7.2 \\
\hline $1911 \mathrm{~V}$ & Brooks & 0.49 & 6,280 & 33.8 & 90.6 & +14.6 \\
\hline 1920 III & Skjellerup & 1.14 & 5,023 & 22.0 & 90.3 & -7.1 \\
\hline $1921 \mathrm{~V}$ & Reid & 1.63 & 7,999 & 32.4 & 98.0 & -2.0 \\
\hline 1985 II & Shoemaker & 1.21 & 23,930 & 13.9 & 91.1 & -10.5 \\
\hline
\end{tabular}

(c) Kopff 1905 IV, which split. There have been only nine split comets that have periods greater than $10^{4} \mathrm{yr}$ and that have been observed since 1900 .

(d) Wirtanen $1957 \mathrm{VI}$, which also split, at a greater solar distance.

(e) Wolf 1917 III, which, like Bowell, had developed a disk at 5 AU.

The remaining three comets in this category, Bester-Hoffmeister 1959 III, Mrkos 1959 IX, and Rudnick 1967 II, were not as well observed as the others. All eight have aphelia at great enough galactic latitudes to allow the likelihood of perturbation by the galactic density gradient. Therefore, we have no a priori choice as to their origin from a great Oort cloud or from a comet belt far beyond Neptune, but not too far from the ecliptic. On the other hand, if the short-period comets are derived from the latter source, comets such as these eight must provide the criteria for their physical character, if it is different from that of the long-period comets.

\section{Summary}

Comments have been made concerning our understanding of the cometary nucleus as developed in this colloquium. Most of the advances reported earlier have been substantiated, but the mean density of the Halley nucleus may have been underestimated, while the nature of the rotation remains uncertain. The dust/gas ratio apparently needs to be increased by as much as two times, perhaps to unity or higher. CHON grains appear more and more to be important sources of gas. A very low temperature at the formation of comets receives continued support, as does a solar-system composition; a low ${ }^{12} \mathrm{C} /{ }^{13} \mathrm{C}$ ratio may be an exception. Following comments on more than a score of items, this paper has presented problems and data relevant to the origin of comets, particularly the shortperiod comets versus the long-period ones. Evidence has been added to support the thesis that aging among long-period comets increases statistically as the periods decrease. Dis- 
cussion and data followed concerning the orientation of cometary axes with respect to the Galaxy and the properties of clusters defined by these axes. Of particular interest are the clusters or groups related to both the galactic plane and the ecliptic plane.

Clearly, space rendezvous missions or more sophisticated missions to several comets are mandatory if we are to unravel the threads of cometary origin and the relevance of comets to life on the Earth.

\section{Acknowledgment}

I am indebted to NASA's Planetary Geology and Geophysics Program for support of this research.

\section{References}

A'Hearn, M.F., and Millis, R.L. (1980). 'Abundance Correlations Among Comets,' Astron. J., 85, 1528.

A'Hearn, M.F., Schleicher, D.G., Feldman, P.D., Millis, R.L., and Thompson, D.T. (1984). 'Comet Bowell 1980b,' Astron. J., 89, 579.

Belton, M.J.S. (in press). 'Characterization of the Rotation of Cometary Nuclei,' in Comets in the Post-Halley Era, ed. Ray L. Newburn, Jr., Marcia Neugebauer, and Jürgen Rahe, Kluwer Academic Publishers, Dordrecht.

Biermann, L., Huebner, W.F., and Lüst, R. (1983). 'Aphelion Clustering of "New" Comets: Star Tracks Through Oort's Cloud,' Proc. Nat. Acad. Sci. U.S.A., 80, 5151.

Campbell, D.B., Harmon, J.K., and Shapiro, I.I. (1989). 'Radar Observations of Comet Halley,' Astrophys. J., 338, 1094.

Cochran, A.L. (1987). 'Another Look at Abundance Correlations Among Comets,' Astron. J., 92, 231.

Crifo, J.F. (in press). 'Hydrodynamic Models of the Collisional Coma,' in Comets in the Post-Halley Era, ed. Ray L. Newburn, Jr., Marcia Neugebauer, and Jürgen Rahe, Kluwer Academic Publishers, Dordrecht.

Delsemme, A.H. (1986). 'Cometary Evidence of a Solar Companion,' in The Galaxy and the Solar System, ed. R. Smoluchchovski, J.N. Bahcall, and M.S. Mathews, University of Arizona Press, Tucson, p. 173.

Delsemme, A.H. (1987). 'Galactic Tides Affect the Oort Cloud: An Observational Confirmation,' Astron. Astrophys., 187, 913.

Delsemme, A.H. (1988). 'The Chemistry of Comets,' Phil. Trans. Roy. Soc. Lond., A325, 509.

Delsemme, A.H. (in press). 'Nature and History of the Organic Compounds in Comets: An Astrophysical View,' in Comets in the Post-Halley Era, ed. Ray L. Newburn, Jr., Marcia Neugebauer, and Jürgen Rahe, Kluwer Academic Publishers, Dordrecht.

Donn, B. (1977). 'A Comparison of the Compositions of New and Evolved Comets,' in Comets, Asteroids, Meteorites, ed. A.H. Delsemme, University of Toledo Press, Toledo, p. 15.

Duncan, M., Quinn, T., and Tremaine, S. (1990). 'Planetary Perturbations and the Origin of Short-Period Comets,' Astrophys. J., 355, 667. 
Eberhardt, P., Krankowsky, D., Schulte, W., Dolder, U., Lämmerzahl, P., Berthelier, J.J., Woweries, J., Stubbeman, U., Hodges, R.R., Hoffman, J.H., and Illiano, J.M. (1986). 'On the CO and $N_{2}$ Abundance in Comet Halley,' in Proc. 20th ESLAB Symposium, ESA SP-250, Vol. 1, p. 383.

Everhart, E. (1977). 'The Evolution of Comet Orbits as Perturbed by Uranus and Neptune,' in Comets, Asteroids, Meteorites, ed. A.H. Delsemme, University of Toledo, Toledo, p. 99.

Everhart, E., and Marsden, B.G. (1983). 'New Cometary Orbits,' Astron. J., 83, 64. Everhart, E., and Marsden, B.G. (1987). 'Original and Future Orbits III,' Astron. J., 93, 753.

Feldman, P.D. (in press). 'Ultraviolet Spectroscopy of Cometary Comae,' in Comets in the Post-Halley Era, ed. Ray L. Newburn, Jr., Marcia Neugebauer, and Jürgen Rahe, Kluwer Academic Publishers, Dordrecht.

Fernandez, J.A. (1980). 'On the Existence of a Comet Belt Beyond Neptune,' Mon. Not. R. A. S., 192, 481.

Fernandez, J.A., and Ip, W.-H. (1983). 'On the Time Evolution of the Cometary Influx in the Region of the Terrestrial Planets,' Icarus, 54, 377.

Gombosi, T.I. (in press). 'Multidimensional Dusty Gasdynamical Models of Inner Cometary Atmospheres,' in Comets in the Post-Halley Era, ed. Ray L. Newburn, Jr., Marcia Neugebauer, and Jürgen Rahe, Kluwer Academic Publishers, Dordrecht.

Hanner, M.S., and Tokunaga, A.T. (in press). 'Infrared Techniques for Comet Observations,' in Comets in the Post-Halley Era, ed. Ray L. Newburn, Jr., Marcia Neugebauer, and Jürgen Rahe, Kluwer Academic Publishers, Dordrecht.

Harrington, R.S. (1985). 'Implications of the Observed Distributions of Very Long Period Comets,' Icarus, 61, 60.

Heisler, J., and Tremaine, S. (1986). 'The Influence of the Galactic Tidal Field on the Oort Comet Cloud,' Icarus, 65, 13.

Hills, J.G. (1982). 'The Formation of Comets by Radiation Pressure in the Outer Protosun,' Astron. J., 87, 906.

Horanyi, M., and Mendis, D.A. (in press). 'The Electrodynamics of Charged Dust in the Cometary Environment,' in Comets in the Post-Halley Era, ed. Ray L. Newburn, Jr., Marcia Neugebauer, and Jürgen Rahe, Kluwer Academic Publishers, Dordrecht.

Huebner, W.F., Boice, D.C., Schmidt, H.U., and Wegmann, R. (in press). 'Structure of the Coma: Chemistry and Solar Wind Interaction,' in Comets in the Post-Halley Era, ed. Ray L. Newburn, Jr., Marcia Neugebauer, and Jürgen Rahe, Kluwer Academic Publishers, Dordrecht.

Jackson, W.M. (in press). 'Recent Laboratory Photochemical Studies and Their Relationship to the Photochemical Formation of Cometary Radicals,' in Comets in the PostHalley Era, ed. Ray L. Newburn, Jr., Marcia Neugebauer, and Jürgen Rahe, Kluwer Academic Publishers, Dordrecht.

Jessberger, E.K., and Kissel, J. (in press). 'Chemical Properties of Cometary Dust and a Note on Carbon Isotopes,' in Comets in the Post-Halley Era, ed. Ray L. Newburn, Jr., Marcia Neugebauer, and Jürgen Rahe, Kluwer Academic Publishers, Dordrecht. Jewitt, D. (in press). 'Cometary Photometry,' in Comets in the Post-Halley Era, ed. Ray L. Newburn, Jr., Marcia Neugebauer, and Jürgen Rahe, Kluwer Academic Publishers, Dordrecht. 
Klinger, J. (in press). 'Physical Properties of Frozen Volatiles-Their Relevance to the Study of Comet Nuclei,' in Comets in the Post-Halley Era, ed. Ray L. Newburn, Jr., Marcia Neugebauer, and Jürgen Rahe, Kluwer Academic Publishers, Dordrecht.

Kresak, L. (1973). 'Short-Period Comets at Large Heliocentric Distances,' Bull. Astr. Inst. Czech., 24, 264.

Kresak, L. (1977). 'On the Differences Between the New and Old Comets,' Bull. Astr. Inst. Czech., 28, 346.

Kresak, L. (1987). 'Aging of Comets and Their Evolution in Asteroids in the Evolution of the Small Bodies of the Solar System,' Soc. Italiana di Fisica, Bologna, 202.

Lüst, R. (1984). 'The Distribution of the Aphelion Directions of Long-Period Comets,' Astron. Astrophys., 141, 94.

Marsden, B.G. (1986). Catalogue of Cometary Orbits, 5th ed., Smithsonian Astrophysical Observatory.

Marsden, B.G., and Sekanina, Z. (1973). 'On the Distribution of "Original" Orbits of Comets of Large Perihelion Distance,' Astron. J., 78, 1118.

Marsden, B.G., Sekanina, Z., and Everhart, E. (1978). 'New Osculating Orbits for 110 Comets and Analysis of Original Orbits for 200 Comets,' Astron. J., 83, 64.

McDonnell, J.A.M., Lamy, P.L., and Pankiewicz, G.S. (in press). 'Physical Properties of Cometary Dust,' in Comets in the Post-Halley Era, ed. Ray L. Newburn, Jr., Marcia Neugebauer, and Jürgen Rahe, Kluwer Academic Publishers, Dordrecht.

Meech, K.J. (in press). 'Physical Aging in Comets,' in Comets in the Post-Halley Era, ed. Ray L. Newburn, Jr., Marcia Neugebauer, and Jürgen Rahe, Kluwer Academic Publishers, Dordrecht.

Meisel, D.D., and Morris, C.S. (1976). 'Comet Brightness Parameters: Definition, Determination and Correlations,' in The Study of Comets, Pt. 1, NASA SP-393, p. 410.

Morris, D.E., and Muller, R.A. (1986). 'Tidal Gravitational Forces: The Infall of "New" Comets and Comet Showers,' Icarus, 65, 1.

Mukai, T. (1989). Paper presented at the "Comets in the Post-Halley Era" colloquium held in Bamberg, Federal Republic of Germany, April 24-28, 1989.

Newburn, R.L., Jr., and Spinrad, H. (1984). 'Spectrophotometry of Seventeen Comets I.,' Astron. J., 89, 289.

Newburn, R.L., Jr., and Spinrad, H. (1985). 'Spectrophotometry of Seventeen Comets II.,' Astron. J., 90, 2581.

Newburn, R.L., Jr., and Spinrad, H. (1989). 'Spectrophotometry of 25 Comets: PostHalley Updates for 17 Comets Plus New Observations for Eight Additional Comets,' Astron. J., 97, 552.

Oort, J.H. (1950). 'The Structure of the Cloud of Comets Surrounding the Solar System and a Hypothesis Concerning Its Origin,' Bull. Astr. Inst. Neth., 11, 91.

Oort, J.H., and Schmidt, M. (1951). 'Differences Between New and Old Comets,' Bull. Astr. Inst. Neth., 11, 259.

Sekanina, Z. (1982). 'Comet Bowell (1980b): An Active-Looking Dormant Object?', Astron. J., 87, 161.

Szegö, K. (in press). 'P/Halley, the Model Comet, in View of the Imaging Experiment Aboard the VEGA Spacecraft,' in Comets in the Post-Halley Era, ed. Ray L. Newburn, Jr., Marcia Neugebauer, and Jürgen Rahe, Kluwer Academic Publishers, Dordrecht. 
Vanysek, V. (in press). 'Isotopic Ratios in Comets,' in Comets in the Post-Halley Era, ed. Ray L. Newburn, Jr., Marcia Neugebauer, and Jürgen Rahe, Kluwer Academic Publishers, Dordrecht.

Vsekhsvyatskii, S.K. (1964). 'Physical Characteristics of Comets,' Trans. from Russian, Israel Program, Jerusalem.

Weaver, H.A., Mumma, M.J., and Larson, H.P. (in press). 'Infrared Spectroscopy of Cometary Parent Molecules,' in Comets in the Post-Halley Era, ed. Ray L. Newburn, Jr., Marcia Neugebauer, and Jürgen Rahe, Kluwer Academic Publishers, Dordrecht.

Weaver, H.A., Feldman, P.D., Festou, M.C., A'Hearn, M.F., and Keller, H.U. (1981). 'IUE Observations of Faint Comets,' Icarus, 47, 449.

West, R.M. (1990). 'Post-Perihelion Observations of Comet P/Halley II. $r=10.1$ AU,' Astron. Astrophys., 228, 531.

Whipple, F.L. (1978). 'Cometary Brightness Variation and Nucleus Structure,' The Moon and Planets, 18, 343.

Whipple, F.L. (1989). 'Comets in the Space Age,' Astrophys. J., 341, 1.

Yeomans, D.K. (in press). 'Cometary Orbital Dynamics and Astrometry,' in Comets in the Post-Halley Era, ed. Ray L. Newburn, Jr., Marcia Neugebauer, and Jürgen Rahe, Kluwer Academic Publishers, Dordrecht. 